Proyecciones Journal of Mathematics

Vol. 29, No 2, pp. 193-199, December 2010.

Universidad Católica del Norte

Antofagasta - Chile

\title{
PARTIAL ORDERS IN REGULAR SEMIGROUPS
}

\author{
K. V.R. SRINIVAS \\ REGENCY INSTITUTE OF TECHNOLOGY, INDIA \\ $A N D$ \\ Y. L. ANASUYA \\ ANDHRA UNIVERSITY, INDIA \\ Received : April 2009. Accepted : September 2010
}

\begin{abstract}
First we have obtained equivalent conditions for a regular semigroup and is equivalent to $N=N 1$ It is observed that every regular semigroup is weakly separative and $C \subseteq S$ and on a completely regular semigroup $S \subseteq N$ and $S$ is partial order. It is also obtained that a band $(S,$.$) is normal iff C=N$. It is also observed that on a completely regular semigroup $(S,),. C=S=N$ iff $(S,$.$) is locally$ inverse semigroup and the restriction of $C$ to $E(S)$ is the usual partial order on $E(S)$. Finally it is obtained that, if $(S,$.$) is a normal band$ of groups then $C=S=N$.
\end{abstract}

Key Words : Locally inverse semigroup, orthodox semigroup, completely regular semigroup, normal band.

AMS Subject Classification No. : $20 M 18$. 


\section{Introduction}

On an arbitrary semigroup $(S,$.$) . Let us consider the following relations$ $C, N, N 1$ and $S$, defined for all $a, b \in S$ :

$(a, b) \in C \Longleftrightarrow a s a=a s b=b s a$ for all $s \in S$,

$(a, b) \in N \Longleftrightarrow a=a x a=a x b=b x a$ for some $x \in S$,

$(a, b) \in N_{1} \Longleftrightarrow a=x a=x b=$ by for some $x, y \in S$,

$(a, b) \in S \Longleftrightarrow a^{2}=a b=b a$.

Obviously, $N \subseteq N 1$, and easy calculations show that $N 1$ is a partial order on $S$ (cf. [6]).

The relation $C$ was defined in [1] by Conrad, and it was proved in [2] by Burgess and Raphael (cf. Lemma 1 ) that $\mathrm{C}$ is a partial order on $\mathrm{S}$ iff $(\mathrm{S},$.$) is weakly separative, i. e., for all a, b \in S$

$$
a s a=a s b=b s a=b s b \text { foralls } \in \text { Simplies } a=b .
$$

In [3], Nambooripad defined $N$ and showed that it is a partial order iff $(S,$.$) is regular (cf. Lemma 2). The relation S$ was introduced by Drazin in [4], and he proved that, for any completely regular semigroup, $S$ is a partial order on $S$, and

$$
C \subseteq S \subseteq N
$$

holds (cf. Lemma 3). Finally, for any regular semigroup $(S,$.$) , the natural$ partial order $\leq$

$$
a \leq b \Longleftrightarrow a=b e=\text { fbforsomee, } f \in E(S)
$$

satisfies $\mathrm{N} \subseteq \leq$.

It was shown in [5] that for any completely regular semigroup $(S,$.$) the$ relations $S$ and $\leq$ coincide iff $S$ is a cryptogroup, i. e., Greens H-relation is a congruence on $(S,$.$) , and that C$ and $S$ coincide iff $S$ is a normal cryptogroup, i. e., $S / H$ is a normal band.

Lemma 1. The following statements are equivalent. a) $(S,$.$) is weakly$ separative. b) $C$ is a partial order. c) $C$ is anti-symmetric.

Proof. Since $C$ is reflexive, and $b) \Rightarrow c$ ) as well as $c) \Longleftrightarrow a$ ) are obvious, we have to prove that a) implies the transitivity of $C$. So assume $(a, b),(b, c) \in$ $C$. Then $(a s a) t(a s c)=a s a t b s c=a s a t b s b=a s a t a s b=(a s a) t(a s a)=$ $a s b t a s a=a s b t b s a=a s c t b s a=(a s c) t(a s a)$ for all $s, t \in S$ yields $a s a=$ ascbya). Similarly one gets asa $=c s a$, hence $(a, c) \in C$. 
Lemma 2. (cf. [5], Propositions 3 and 4) The following statements are equivalent.

a) $(S,$.$) is regular.$

b) $N_{1} \subseteq N$.

c) $N_{1}=N$.

d) $\leq \subseteq N$.

e) $\leq$ and $N$ coincide.

f) $N$ is a partial order.

g) $N$ is reflexive.

Proof. We only have to show $a) \Rightarrow b$ ) and $a) \Rightarrow d$ ), since $b) \Rightarrow c$ ), $c) \Rightarrow f(, d) \Rightarrow e), e) \Rightarrow f(f) \Rightarrow g$ ) and $g) \Rightarrow a$ ) are trivial. So first assume $(a, b) \in$ for $a \neq b$ in $S$. Then there are $x^{\prime}, x, y \in S$ such that $a=a x^{\prime}$ aand $a=x a=x b=b y$. Then $a\left(y x^{\prime} x\right) a=x b\left(y x^{\prime} x a\right)=x a x^{\prime} x a=$ $a x^{\prime} a, a\left(y x^{\prime} x\right) b=x b y x^{\prime} x b=x a x^{\prime} a=a x^{\prime} a=a$ and $b\left(y x^{\prime} x\right) a=a x^{\prime} a=a$ show $(a, b) \in N$. Now assume $a \leq b$ in $S$. Then there are $e, f \in E(S)$ and $y \in S$ such that $a=b e=f b$ and $b=b y b$. Then $a=f b=f b y b=a y b$, $\mathrm{a}=\mathrm{be}=$ bybe=byaandaya $=\mathrm{f}$ bya $=\mathrm{f} \mathrm{a}=\mathrm{f} \mathrm{f} \mathrm{b}=\mathrm{f} \mathrm{b}=$ ashowthat $(\mathrm{a}, \mathrm{b})$ $\in N$.

Lemma 3. For any semigroup $(S,$.$) the following statements hold. a) If$ $(S,$.$) is regular, then it is weakly separative. b) If (S,$.$) is regular then$ $C \subseteq S$. c) If $(S,$.$) is completely regular then S \subseteq N$ and $S$ is a partial order.

Proof. a) Consider $a, b \in S$ such that $a s a=a s b=b s a=b s b$ for all $\mathrm{s} \in$ S.Thenfor $x, y \in$ Ssuchthata $=$ axa, $\mathrm{b}=$ bybonehasa $=$ axa $=$ bxb $=($ byb $) \mathrm{x}($ byb $)=$ byaxayb $=$ byayb $=\mathrm{b} \cdot b)$ For $(\mathrm{a}, \mathrm{b}) \in C$, there is some $x \in S$ such that $a=a x a$. This implies $a^{2}=a x a a=a x a b=a b$ and $a^{2}=a a x a=b a x a=b a$, hence $(a, b) \in S$. c) For $(a, b) \in S$ one has $a 2=a b=b a$. Since $(S,$.$) is completely regular, there is x \in S$ such that $a=a x a$ and $a x=x a$. Then $a=a x a=a a x=b a x=b x a$ and $a=a x a=$ $x a a=x a b=a x b$ show $(a, b) \in N$. Clearly, $S$ is reflweexive. For $(\mathrm{a}, \mathrm{b})$, $(\mathrm{b}, \mathrm{a}) \in S \subseteq N$, onehasa $=$ bsinceNisanti - symmetric.For $(a, b),(b, c) \in$ Sanda $=$ axa,$a x=x a$ forsomex $\in$ Swehavea $=a 2 x=$ baxandthusca $=$ $c b a x=b 2 a x=b a 2 x=b a=a 2 a n d s i m i l a r l y a c=a 2$, whichshows $(a, c) \in$ $S$.

Recall from [8], p. 222, that a regular semigroup $(S,$.$) in which the set$ of idempotents $E(S)$ is a subsemigroup is called orthodox, and if $E(S)$ is a normal band then $(S,$.$) is called a generalized inverse semigroup. Moreover,$ the class of generalized inverse semigroups is the intersection of the class 
of all orthodox semigroups and the class of all locally inverse semigroups. For the latter we have the following characterization (due to Lemma 2 and [9], IX. Proposition 3.2):

Theorem 4. Let $(S,$.$) be a regular semigroup. Then the following state-$ ments are equivalent: a) $(S,$.$) is locally inverse; b) N$ is compatible; c) If $a N b$ and $b^{\prime}$ is an inverse of $b$, then there is a unique inverse $a^{\prime}$ of a such that $a^{\prime} N b^{\prime}$.

In [10], the following lemma was proved.

Lemma 5 : Let $(S,$.$) be a locally inverse semigroup and \leq$ a partial order on $S$ with the following properties: (i) $\leq$ is compatible with the multiplication; (ii) the restriction of $\leq$ on $E(S)$ coincides with the usual partial order on $E(S)$; (iii) for $a \leq b$ and any inverse $b^{\prime}$ of $b$ there is a unique inverse $a^{\prime}$ of a such that $a^{\prime} \leq b^{\prime}$. Then $\leq^{\prime}$ coincides with $N$.

Proof : First we observe the following ([10], Lemma 1$)$ Let $\leq$ be a partial order on a regular semigroup which is compatible with multiplication and whose restriction to $E(S)$ is the usual partial order on $E(S)$. Further, if it satisfies the property $a b$ and $b^{\prime}$ is an inverse of $b$ then a unique inverse $a^{\prime}$ exists such that $a^{\prime} b^{\prime}$. Then $a \in S$ and $a \leq e \in E(S)$ imply that a is an idempotent $\rightarrow(1)$.

Let $\leq^{\prime}$ be a partial order on a regular semigroup $\mathrm{S}$ which is compatible with multiplication and whose restriction to $E(S)$ is the usual partial order on $E(S)$, then Nambooripads partial order $N \subseteq \leq$, as let $a N b$ so that or any inverse $b^{\prime}$ of $b$, we have $a b^{\prime} N b b^{\prime}$, then $b b^{\prime} \in E(S)$. Further $a b^{\prime} \in E(S)$ [3] and hence $a b^{\prime} N b b^{\prime} a n d \mathrm{ab}^{\prime} b N b b^{\prime} b=b$. since $a N b, a=e b$ for some idempotent $e \in R a$ and hence $e b b^{\prime} b \leq^{\prime} b$, so that $a=e b \leq^{\prime} b$ and hence $N \subseteq \leq^{\prime} \rightarrow(2)$. Now, let $a \leq^{\prime} b$ so that $a b^{\prime} \leq b b^{\prime}$ where $b^{\prime}$ is an inverse of $b$.

Since $b b^{\prime} \in E(S)$ we have $a b^{\prime} \in E(S)$ by using (1) so that $a b^{\prime} \leq^{\prime} b b^{\prime}$. Similarly $b^{\prime} a \leq^{\prime} b^{\prime} b$. And also $a \leq^{\prime} b$, by assumption here exist a unique inverse a'ofasuchthata ${ }^{\prime} \leq^{\prime} b^{\prime}$ and hence $a a^{\prime} \leq^{\prime} b b^{\prime}$ and $a^{\prime} a \leq^{\prime} b^{\prime} b$ (since $\leq^{\prime}$ is compatible with multiplication). Hence, $a a^{\prime} N b b^{\prime}$ and $a^{\prime} a N b^{\prime} b$. Now, $a=a a^{\prime} a N a b^{\prime} b=b b^{\prime} b^{\prime} b\left(\right.$ as $\left.a b^{\prime}=b b^{\prime} a b^{\prime}\right)=b\left(b^{\prime} a\right)\left(b^{\prime} b\right)=b b^{\prime} N b$ imply that $a N b$ so that $\leq^{\prime} \subseteq N \rightarrow(3)$. From (2) and $(3) \leq^{\prime}=N$.

For any band $(S,$.$) , clearly an orthodox semigroup, the relations S, N$ and $N$ coincide with $\leq$, and one obviously has $C \subseteq N$.

Lemma 6. For a band $(S,$.$) the following statements are equivalent. a)$ $(S,$.$) is normal, i. e., axya = ayxa for all a, x, y \in S$. b) $N \subseteq C$. c) $C=N$

Proof. $a) \Rightarrow b)$ : For $a \leq b$ in $S$, i. e., $a=a b=b a$, one has $a s b=b a s b=$ $b s a b=b s a$ for all $s \in S$ and hence $a s a=a s a b=b s a a=b s a=a s b$. This 
shows $(a, b) \in C$. b) $\Rightarrow c)$ is clear. c) $\Rightarrow a)$ : Since $C$ is compatible for any semigroup, the result follows from Theorem 4 and the remark before it.

Corollary 7. Let $(S,$.$) be an orthodox semigroup. If N$ and $C$ coincide on $E(S)$, then $E(S)$ is a normal band.

Definition 8. A semigroup $(S,$.$) is called separative if for all a, b \in S$

$$
\begin{gathered}
(i) a^{2}=a b, b a=b^{2} \Rightarrow a=b, \text { and } \\
(i i) a^{2}=b a, a b=b^{2} \Rightarrow a=b,
\end{gathered}
$$

and $(S,$.$) is called quasi separative if$

$$
(\text { iii }) a^{2}=a b=b a=b^{2} \Rightarrow a=b .
$$

Note that every left or right cancellative semigroup is separative, and that every separative semigroup is quasi separative. Moreover, every completely regular semigroup is quasi separative, as was shown in the proof of Lemma $3 \mathrm{c}$ ).

Proposition 9. For any quasi separative semigroup $(S,$.$) the following$ statements hold. a) $\mathrm{C} \subseteq S . b) \operatorname{If}(\mathrm{ab}) 2=\mathrm{a} 2 \mathrm{~b} 2$ foralla, $\mathrm{b} \in S$, then $\mathrm{S}$ is a partial order on $S$.

Proof. a) For $(a, b) \in C$ one has $a s a=a s b=b s a$ for all $s \in S$. Putting $s=$ $a$, one gets $a 3=a 2 b=b a 2$, from which $a 4=a 2(b a)=b a 3=(b a) a 2$ follows. Putting $s=a b$ yields $a 2(b a)=a 2 b 2=(b a) 2$, hence $a 4=a 2(b a)=(b a) a 2=$ (ba)2. Since $(S,$.$) is quasi separative, this implies a 2=b a$. Putting $s=b a$ yields $(a b) a 2=(a b) 2=b 2 a 2$, hence $a 4=(a b) a 2=a 2(a b)=(a b) 2$. Again by separativity, one gets $a 2=a b$.

This shows $C \subseteq S$. b) Clearly, $S$ is always reflexive, and it is antisymmetric if and only if $(\mathrm{S}$, . ) is quasi separative. To show transitivity, take some $(\mathrm{a}, \mathrm{b}),(\mathrm{b}, \mathrm{c}) \in S$. Then $a 2=a b=b a$ and $b 2=b c=c b$ imply $a 4=(a b) 2=a 2 b 2=a 2 b c=a 3 c=a 2(a c)$ as well as $a 4=a b 2 a=a c b a=$ $(a c) a 2$ and $(a c) 2=a 2 c 2=a b c 2=a b 2 c=a 2 b c=a 2 b 2=a 4$.

From $a 4=(a c) a 2=a 2(a c)=(a c) 2$ follows $a 2=a c$, since $(S,$.$) is quasi$ separative. Similarly, one gets $a 2=c a$ and therefore $(a, c) \in S$.

Proposition 10. For a completely regular semigroup $(S,$.$) the following$ conditions are equivalent: a) $C=S=N$; b) $(S,$.$) is locally inverse and$ the restriction of $C$ to $E(S)$ is the usual partial order on $E(S)$. 
Proof. a) $\Rightarrow b)$ : Since $C$ is compatible for any semigroup, it follows from Theorem $4 \mathrm{~b})$. b) $\Rightarrow a)$ : For $(a, b) \in C$ and any inverse $b^{\prime} \in S$ of $b$, by Theorem $4 \mathrm{c}$ ) there is a unique inverse $a^{\prime} \in S$ of a such that $a^{\prime} N b^{\prime}$. Now Theorem $4 \mathrm{~b}$ ) implies $a^{\prime} b N b^{\prime} b$ and since $b^{\prime} b$ is an idempotent in the regular semigroup $(S,$.$) we get a^{\prime} b \in E(S)$. This yields $a^{\prime} b C b^{\prime} b$. Similarly $b a^{\prime} C b b^{\prime}$ follows.

The compatibility and transitivity of $C$ now imply $a^{\prime}=a^{\prime} a a^{\prime} C a^{\prime} b a^{\prime}=$ $a^{\prime} b b^{\prime} b a^{\prime} C b^{\prime} b b^{\prime} b a^{\prime}=b^{\prime} b a^{\prime} C b^{\prime} b b^{\prime}=b^{\prime} . B y L e m m a 3, \mathrm{C} \subseteq N$ such that any inverse $a^{\prime}$ of a which satisfies $a^{\prime} C b^{\prime}$ is uniquely determine by $b^{\prime}$. Now Lemma 5 implies $\mathrm{C}=\mathrm{N}$.

Proposition 11. If $(S,$.$) is a normal band of groups then C=S=N 4$.

Proof. Let $(\mathrm{S},$.$) be a normal band of groups then S$ is completely regular and orthodox and also $\mathrm{H}$ is congruence [7, Prop. 1.7, Page 106].

With respect to Lemma 3 we have to show $N \subseteq C$. So assume $(a, b) \in$ $N$. Hence there are $e, f \in E(S)$ such that $a=b e=f b$. For $s \in S$ and $x=a s a=b e s b e, y=a s b=b e s b$ and $z=b s a=b s b e$ we have to show $x=y=z$. Note that in any regular semigroup $x x^{\prime}=y y^{\prime}, y^{\prime} x=y^{\prime} y$ for inverses $x^{\prime}, y^{\prime}$ of $x$ and $y$, respectively imply $x=y$, since $x=x x^{\prime} x=$ y $\mathrm{y}^{\prime} x=y y^{\prime} y=y$. Since $S$ is the union of groups, we may assume that $a \in H g, b \in H h$ and $s \in H k$.

Now consider $x x^{\prime}=($ besbe $)(\text { besbe })^{\prime}=$ besbeeb $^{\prime} s^{\prime} e b^{\prime}$ (since $S$ is orthodox) $=$ besbeb's $s^{\prime} b^{\prime} \in$ Hhekhehkeh $=$ Hhekheh(normality $)=$ Hhkeh.y $y^{\prime}=$ $($ besb $)(\text { besb })^{\prime}=$ besbb $s^{\prime} e b^{\prime} \in$ Hhekhkeh(normality $)=$ Hhkeh.

Since $x x^{\prime}, y y^{\prime}$ are idempotents and $x x^{\prime}, y y^{\prime} \in$ Hhkeh, Hence $x x^{\prime}=y y^{\prime}$. Consider $y^{\prime} x=(\text { besb })^{\prime}$ besbe $=b^{\prime} s^{\prime} e\left(b^{\prime} b\right) e s b e=b^{\prime} s^{\prime} b^{\prime}$ besbe $\in$ Hhkehehkh $=$ Hhkehkehh (normality) $=H h k e h$. Similarly $y^{\prime} y \in H h k e h$. Since $y^{\prime} x, y^{\prime} y$ are idempotents and $y^{\prime} x, y^{\prime} y \in H h k e h$. Hence, $y^{\prime} x=y^{\prime} y$. Since $x x^{\prime}=y y^{\prime}$ and $y^{\prime} x=y^{\prime} y$ imply that $x=y$. Hence, $a s a=a s b$, for all $s \in S$. Similarly $a s b=b s a$, for all $s \in S$. Hence, $(a, b) \in C$ so that $C=N$.

\section{References}

[1] Conrad, P. F., The hulls of semiprime rings, Bull. Austral. Math. Soc. 12, pp. 311314(1975).

[2] Burgess, W. D., Raphael, R., On Conrads partial order relation on semiprime rings and semigroups, Semigroup Forum 16, pp. 133140, (1978). 
[3] Nambooripad, K. S. S., The natural partial order on a regular semigroup, Proc. Edinburgh Math. Soc. 23, pp. 249260, (1980).

[4] Drazin, M. P., A partial order in completely regular semigroups, J. Algebra 98, pp. 362374, (1986).

[5] Liu, Guo-Xin, Song, Guang-tian, Some partial orders on completely regular semigroups, J. Univ. Sci. and Tech. China 34, No. 5, pp. 524528, (2004).

[6] Petrich, M., Reilly, N., Completely Regular Semigroups, Wiley Sons, New York, (1999).

[7] Petrich, M., Introduction to Semigroups, Charles E. Merrill Publ. Comp., Columbus, Ohio, (1973).

[8] Howie, J. M., Fundamentals of Semigroup Theory, Clarendon Press, Oxford (1995).

[9] Grillet, P. A., Semigroups. An Introduction to the Structure Theory, Pure and Applied Mathematics 193, Marcel Dekker, New York (1995).

[10] Ramana Murthy, P. V., Srinivas, K. V. R., Characterization of partial orders on regular semigroups, A. P. Akademi of sciences, Hyderabad, Vol. 8, No. 4, pp. 289 292, (2004).

\author{
K. V. R. Srinivas \\ Regency institute of technology, \\ YANAM - 533464, \\ Near Kakinada \\ e-mail : srinivas_kandarpa06@yahoo.co.in \\ and
}

\title{
Y. L. Anasuya
}

Department of Mathematics

Andhra University

Visakhapatnam

A. U. VIZAG - 533003

INDIA

e-mail : anasuyapamarthy@gmail.com 